\title{
The physiological benefits of paraplegic orthotically aided walking
}

\author{
C Ogilvie MD FRCS, ${ }^{1}$ P Bowker PhD,${ }^{2}$ D I Rowley MD BMedBiol FRCS (Ed) ${ }^{3}$
}

Hope Hospital, Salford, England. ' Senior Orthopaedic Registrar, Bristol Children's Hospital, Bristol, England; ${ }^{2}$ Senior Lecturer in Orthopaedic Mechanics, Salford University, Salford, England; ${ }^{3}$ Professor of Orthopaedics, Dundee University, Dundee, Scotland.

\begin{abstract}
A group of first-time paraplegic users of a particular ambulatory orthosis was studied to assess the physiological benefits of changing from a wholly sedentary lifestyle to one which included regular periods of upright weight-bearing and walking. Little clinically apparent benefit was noted in respiratory mechanics. There was a subjective reduction in urinary tract infections, but without a corresponding reduction in the level of bacteria. A possible improvement in bone density was noted in a small subgroup. It is concluded that, at least in the shorter term, the physiological benefits to paraplegics of using walking orthoses are not as great as has generally been claimed in the literature.
\end{abstract}

Keywords: orthosis; walking; paraplegia; physiological benefits.

\section{Introduction}

The potential physiological benefits to the paraplegic of being able to stand and achieve some mobility in the upright posture have been acknowledged for many years. ${ }^{1,2}$ Since that time many workers in the field have repeated the view that the vertical, weight-bearing posture bestows on the paraplegic improvements in respiratory mechanics, urological drainage, bone density and lower limb blood flow. Scientific data supporting this view are however relatively limited. Respiratory mechanics have been reported to improve in children using the swivel walker, ${ }^{3}$ whilst greater bone density is seen in the less handicapped, and consequently more mobile, children with spina bifida. ${ }^{4}$ Similarly, there were few fractures in a spina bifida group who had been part of a walking programme when compared with a group who had been prescribed a wheelchair in early life. ${ }^{5}$

Thus, in order to examine and quantify these potential benefits, a group of previously chairbound paraplegic subjects, who were regaining or commencing weight-bearing for the first time in an ambulatory

Correspondence: C Ogilvie, Consultant Orthopaedic Surgeon, Department of Orthopaedics, Musgrove Park Hospital, Taunton, Somerset. orthosis, were subjected to a series of standard physiological tests to measure respiratory mechanics, urological drainage, bone density and lower limb vascularity. The orthosis employed was the reciprocating gait orthosis, ${ }^{6,7}$ a combined trunk and leg brace which facilitates ambulation through reciprocal motion at the hips which is controlled by Bowden cables interlinking the two joints. Extrinsic stability and energy transfer are achieved via a walking frame or crutches. We have previously reported our data on lower limb blood flow: ${ }^{8}$ this paper presents the results of the remaining three physiological tests.

\section{Patients}

A group of 13 patients who had been accepted for fitting with a reciprocating gait orthosis (RGO) according to the prescription guidelines in Table I were recruited. During the course of the study 2 patients subsequently declined to continue, leaving a group of 6 male and 5 female patients with an age range of 2-42 years. Table II shows the age grouping and diagnoses of these patients. All had been chairbound for at least one year, and had not been using any other standing device, though they may have previously attempted the use of other 
Table I Prescription criteria for the reciprocating gait orthosis

Triceps: MRC power 4

Pelvic obliquity $<10 \mathrm{deg}$

Hip or knee flexion contractures $10 \mathrm{deg}$ maximum

Ankle equinus $10 \mathrm{deg}$ maximum

Plantigrade feet

Approximately correct weight for height

Moderate IQ and training potential

Realistic psychological attitude

Table II Age grouping and diagnosis of the patients

\begin{tabular}{lccc}
\hline & Juvenile & Adolescent & Adult \\
\hline $\begin{array}{l}\text { Spina bifida } \\
\text { Spinal injury }\end{array}$ & - & - & 1 \\
$\begin{array}{l}\text { Transverse } \\
\text { myelitis }\end{array}$ & - & 1 & 6 \\
\begin{tabular}{c} 
Spinal tumour \\
\hline
\end{tabular} & - & - & 1 \\
\hline
\end{tabular}

orthoses. In the case of the juvenile patients, this was their first 'walking orthosis'. They had no medical problems other than those related to their paraplegia. A small group of 3 chairbound patients who were unsuitable for the reciprocating gait orthosis was also recruited.

\section{Procedures}

Patients in both the study and control groups were subjected to a series of physiological tests to examine their respiratory function, urological drainage and bone density. The tests were repeated at 6 monthly intervals from the subject's first referral through the period of fitting and training in the orthosis to the time when they were independent and regular ambulators, an average of 5 months, when the RGO was used on average daily for a period of 3 hours. Follow up was for a mean of 24 months (range 18-30 months).

Respiratory mechanics testing was carried out using a standard spirometer (Micromedical Pocket Spirometer) which produced a read-out of functional vital capacity (FVC), peak expiratory flow rate (PEFR), and forced expiratory volume in one second (FEV1). The expected figures for each patient based on arm span measurements, were obtained from standard tables. ${ }^{9}$ One of the juvenile orthosis users was unable to cooperate with this procedure.

Urological drainage was assessed indirectly using three approaches. Firstly a midstream specimen of urine was cultured and a total colony count obtained at 48 hours. Secondly an Amercan Monitor Parallel Analyser was used to measure blood urea and electrolytes, including calcium, phosphate and alkaline phosphatase, the latter to give an indication of bone turnover. Thirdly, the renal tract was examined ultrasonically. Ultrasound was chosen in preference to radiology in view of the large number of xrays that would have been required. Additionally it has been shown to be an accurate method of diagnosing bladder residual volumes, calculi, hydronephrosis and hydroureter, ${ }^{10-12}$ as well as being painless and noninvasive.

Bone density was measured using quantitative computerised tomography which is a noninvasive technique with a low radiation dose (100-200 mrem) and a high reproducibility and accuracy. Four patients took part in this aspect of the study. All were traumatic paraplegics, 2 premenopausal women aged 16 and 42 years, and 2 men aged 25 and 28 years. At each scanning session, measurements of bone density were made in the femoral neck and in the lumbar vertebrae. This allowed the patients to act as their own controls as the vertebrae remain weight-bearing when both standing and sitting, whereas the femoral neck carries weight only when standing.

\section{Results}

The means of the respiratory function results for the 10 patients in the study group are presented in Figure 1. The data for the juvenile patient are also shown in the same figure as the lower trace. This patient is shown separately as the volumes are so much smaller than the adults. The control group means are also presented in this figure.

The tests to assess urological drainage showed no clear changes over the investigation period in serum electrolytes or en- 

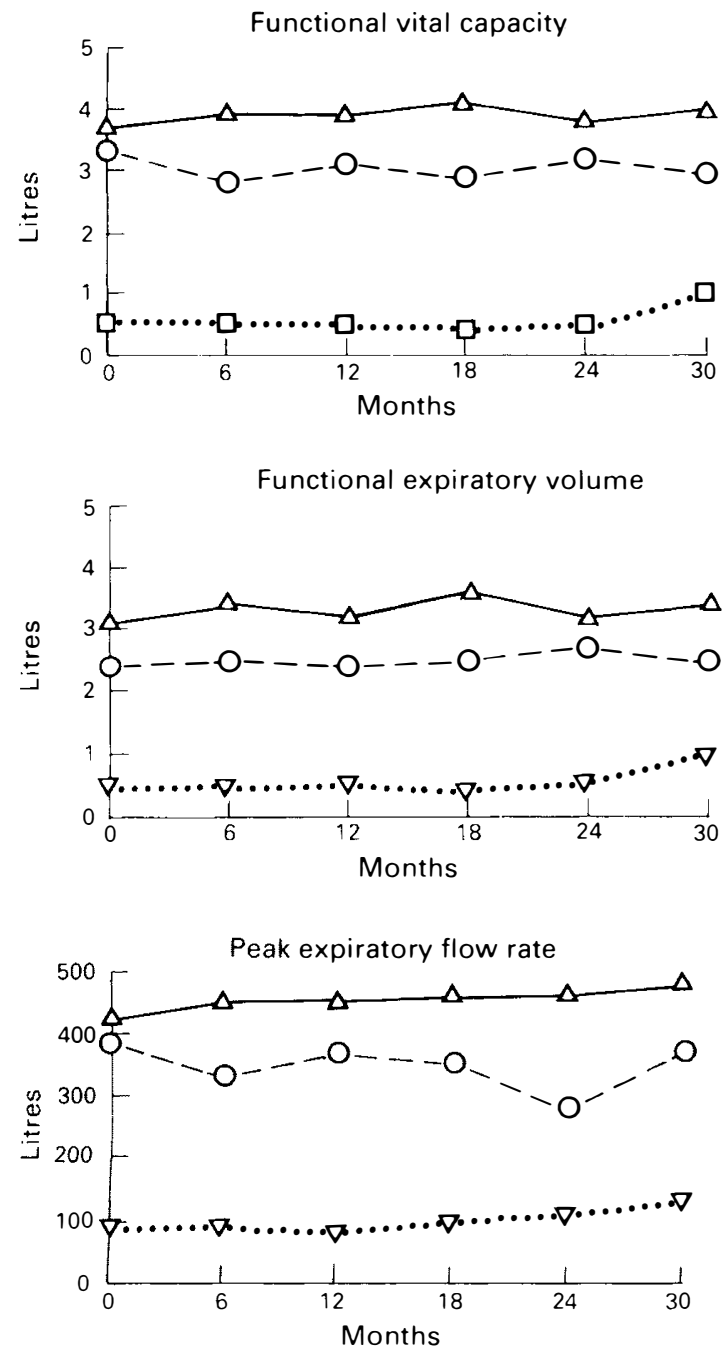

Figure 1 Respiratory mechanics.

zymes, in bacterial colony count or in renal outline or in the ureter. However, an unexpected ovarian cyst was diagnosed and one juvenile patient had a bladder stone identified, both of which were subsequently removed.

The bone density results are shown in Figure 2.

\section{Discussion}

The results of this investigation are overall disappointing. The expected improvements in physiological function did not occur.
The absence of a clear improvement in respiratory mechanics is at variance with the previously published study. ${ }^{2}$ Even in the case of the juvenile patient, who appears to maintain a steady level of respiratory function, there is a decline when the expected increase with growth is taken into account. This improvement may have been hoped for with the removal of the restrictive position of the abdominal contents, preventing diaphragmic excursion. Similarly the use of a walking frame or crutches provides a stable distal 'attachment' for the accessory respiratory muscles to act against. The reason for this failure of enhancement of the respiratory function may be that previously described by Bonnet et al.$^{13}$ They suggested that with a loss of abdominal muscle function, the contents of the abdomen herniate forwards. This decreases the height of the domes of the diaphragm, reducing its excursion and efficiency. This offsets any improvement in the function of the accessory respiratory muscles conferred by the upright posture.

Bone density changes were slightly more encouraging, although the number of patients studied was small. This was partially due to the limited number of patients available and willing to take part in the study and partially due to limited research access to the scanning facility. Bone density would be expected to rise with increased upright activity as has been previously shown. ${ }^{4}$ In the present study 3 of the 4 patients increased or maintained their femoral neck bone density, while their vertebral levels remained relatively unchanged. These 3 patients were using their orthoses for approximately the average time for the whole patient group. The fourth patient had a pressure sore during the study period which restricted use of the RGO, though after a mid-study fall there was an increase, with greater use of the orthosis.

The results presented here suggest that, at least in the shorter term, the physical benefits to paraplegics of using walking orthoses are not as great as previously claimed, the study and control groups results remaining essentially unchanged throughout the investigation period. As all the physiological tests used were standard 


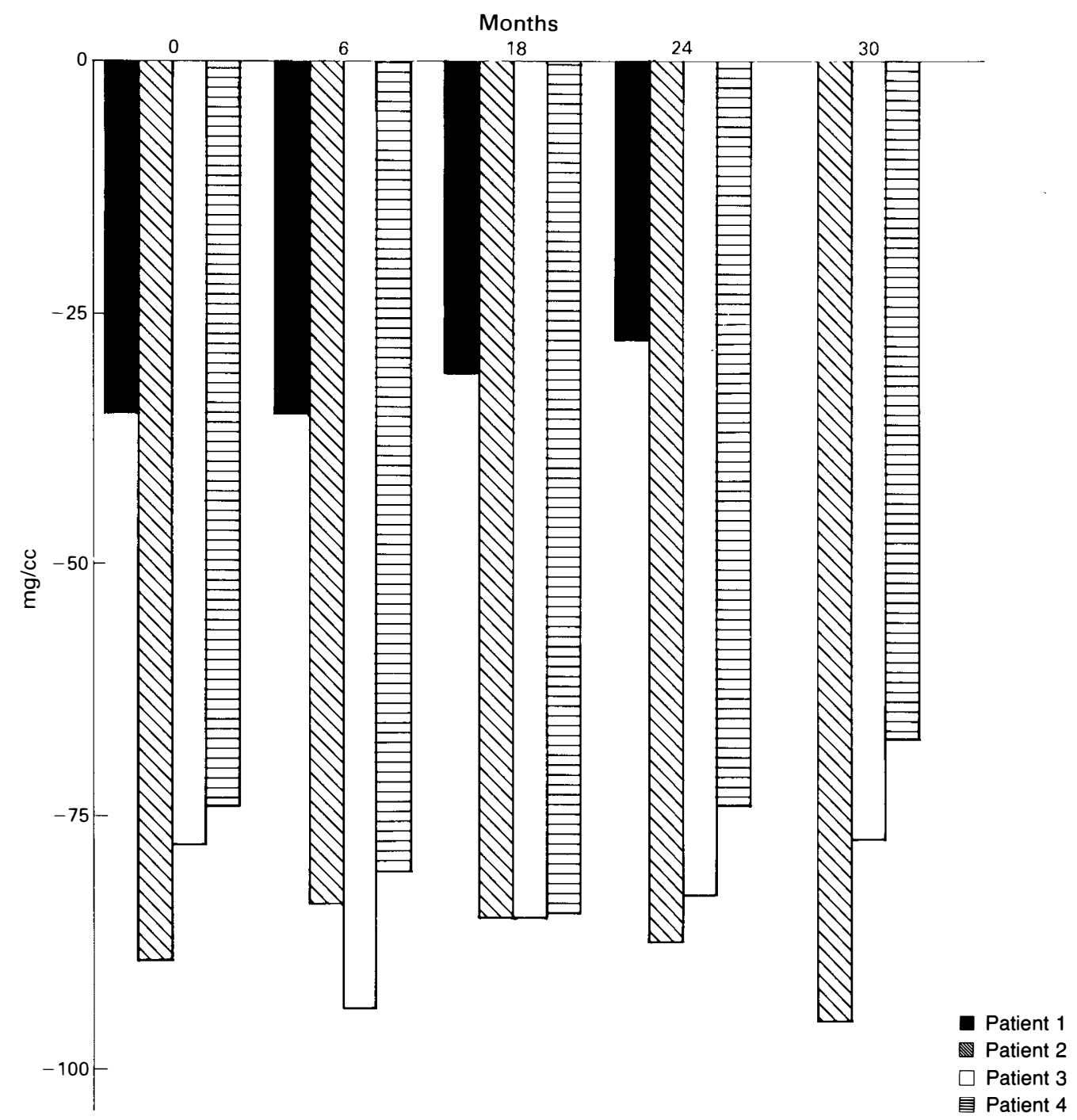

Figure 2 Femoral neck bone density.

clinical tests, any changes of clinical significance in the parameters measured would certainly have been revealed. However, whilst the quantitative data has given a pessimistic view of the physical improvements resulting from the use of a walking orthosis, subjectively the patients were very much more optimistic. In a survey of 30 juvenile and adult users of an RGO, over one third felt that they had enjoyed better general health as a consequence of using the orthosis. This may simply be because a regular interest was taken in their progress, but equally, as most of the patients ultimately used their RGO as an exercise device, may have arisen because they had become generally fitter. These are possibilities which are difficult to quantify.

The results of our investigation indicate that patients who wish to continue to 'walk' for as long as possible, or who wish to attempt to return to 'walking' should not be given the impression that it is the panacea for all their ills. Equally, if the difficulties in 
the use of such an orthosis and the limitathem and they still wish to proceed, the tions in its likely benefits are explained to opportunity should not be denied them.

\section{References}

1 Rose GK, Henshaw JT (1972) A swivel walker for paraplegics: Medical and technical considerations. Biomed Eng 7(9): 420-425.

2 Carroll N (1974) The orthotic management of the spina bifida child. Clin Orth 102: 108-114.

3 Griffiths JC, Henshaw JT, Heywood OB, Zakai ZA (1977) Clinical experience with the swivel walker. Z Kinderchir 22(4): 481-488.

4 Rosenstein D, Greene WB, Herrington LRT, Blum AS (1987) Bone density in meningomyelocele. The effects of ambulatory status. Dev Med Child Neurol 29: 486-494.

5 Mazur J, Shurtleff D, Menelaus M, Colliver J (1989) Orthopaedic management of high level spina bifida. J Bone Joint Surg 71A: 56-61.

6 Yngve DA, Douglas R, Roberts JM (1984) The reciprocating gait orthosis in myelomeningocele. J Ped Orth 4(3): 304-310.

7 Beckman J (1987) The Louisiana State University reciprocating gait orthosis. Physiotherapy 73(8): $386-392$.

8 Messenger N, Bowker P, Rithilia S, Rowley DI, Ogilvie C (1989) The effect of ambulation on blood flow in the paralysed limb. J Biomed Eng 11(5): 249-252.

9 Weng TR, Levinson H (1969) Standards of pulmonary function in children. Am Rev Respir Dis 98: $879-894$.

10 Hederstrom E, Forsberg L (1985) Accuracy of ultrasonography compared with urography in the detection of intrarenal dilation in children. Acta Radiol Diagn 26: 201-207.

11 Kaplan WE (1985) Management of myelomeningocele. Urological Clin NA 12(1): 93-101.

12 Moskowitz PS, Carrol BA, McCoy JM (1980) Ultrasonic volumetrics in children. Radiology 134: 61-64.

13 Bonnet C, Brown JC, Perry J, Nickel VC, Walinski T, Brooks L et al (1975) Evolution of treatment of paralytic scoliosis at Rancho Los Amigos Hospital. J Bone Joint Surg 57 A: 206-215. 